\title{
The Study of the Movement of Cotton Particles and Mathematical Modeling of the Impact of It at the Ginning Point
}

\section{Jamoliddin Ergashev, Khamid Akhmedhodjaev, Abdusamat Karimov, Juramirza Kayumov, Rashida Ergasheva}

Namangan Institute of Engineering and Technology, Namangan, Uzbekistan

Email: ergashev64@mail.ru

How to cite this paper: Ergashev, J., Akhmedhodjaev, K., Karimov, A., Kayumov, J. and Ergasheva, R. (2019) The Study of the Movement of Cotton Particles and Mathematical Modeling of the Impact of It at the Ginning Point. Engineering, 11, 768-776. https://doi.org/10.4236/eng.2019.1111051

Received: August 17, 2019

Accepted: November 12, 2019

Published: November 15, 2019

Copyright $\odot 2019$ by author(s) and Scientific Research Publishing Inc. This work is licensed under the Creative Commons Attribution International License (CC BY 4.0).

http://creativecommons.org/licenses/by/4.0/

\begin{abstract}
This article studied the principles of movements in the horizontal part of the newly constructed ginning camera, which is created by the authors, of the cotton particles. During the movement, the cotton particles get affected by pressing to various surface and forceful turbulent and horizontal movements of the surface. Some foreign mixtures and additional unnecessary objects get separated by the turbulence of the various surfaces and movement of the paneled stripes. Ginning efficiency and the quality of the cotton will be improved as the defects of the cotton particles are removed. "Cotton particle + net surface" movement principle of the Cartesian coordinate system was examined based on the rows of Cartesian coordinate system, by dividing all sides of the system by $m$ weight and has the following second ordered multiple gender differential formula.
\end{abstract}

\section{Keywords}

Cotton Particle, Ginning Process, Ginning Camera, Saw Cylinder, Belt Conveyor, Plank

\section{A Case Study of the Technological Processes in the Working Camera of the Saw Gin}

The primary processing of cotton includes drying, cleaning the raw cotton from large and small foreign matters, ginning, cleaning, moistening and pressing the fiber. The main technological machine of cotton factories for processing medium-fiber varieties of raw cotton is gin, the main task of which is to separate the cotton fiber from the cotton seeds, provided that its natural properties are pre- 
served. Gin, from the day of his invention and practical implementation to the present, has undergone various design changes.

The scientists, designers and workers of the cotton ginning industry have been given the task of improving the quality of the raw cotton being harvested, introducing new more advanced technological machines into the industry, modernizing existing equipment and choosing optimal operating modes, comprehensive mechanization and automation of production.

A. I. Studensev [1] found that the performance of the saw gin depends on the efficiency of the seed output from the raw chamber, and the seed is extracted only from the seed comb, and not along the entire length of the grate (the layer of raw material and seeds moves in the direction from the periphery of the raw chamber to the center). A. I. Studensev correctly asserted that the presence of a large number of bare seeds in a raw roller was impractical. However, his conclusion about the place of seed isolation was not confirmed by the studies of $\mathrm{D}$. A. Kotov and G. I. Boldinsky [2]. The selection of seeds from the raw chamber occurs along the entire length of the cutting arc, but the largest number is in the zone of the seed comb.

M. T. Tillyaev [3] conducted research on the influence of the raw chamber rotation accelerator on the main indicators of the ginning process. He found that: there is a close relationship between gin productivity and the speed of rotation of the raw chamber: with an increase in the speed of the latter, gin productivity increases; with an increase in the speed of rotation of the accelerator to $200 \mathrm{rpm}$, the performance of the gin increases intensively, and then slows down; from the point of view of gin productivity, the optimum rotation speed of the raw roller accelerator is a speed of $200 \mathrm{rpm}$; the use of an accelerator of rotation of the raw chamber leads to a decrease in the residence time of seeds in the raw chamber and, as a result, to a decrease in the content of bare seeds in it; a decrease in the content of bare seeds in the raw chamber leads to an increase in the number of seeds in it with small and large strands of fiber, as well as not ginned cotton particles; an increase in the content in the raw chamber of seeds with small and large strands of fiber leads to an increase in the fiber content of the raw mass, and all this taken together leads to an increase in the productivity of gin.

In the studies of P. N. Tyutin [4], the theoretical foundations of acceleration and stabilization of the rotation of the raw chamber were developed and the parameters of accelerators that contribute to a stable area of their work were determined, and the possibility of combining the processes of acceleration of the raw chamber with additional seed isolation from the central part of the working chamber was found.

In this paper, we investigated the impact of the cotton particles captured by the teeth of a saw on the surface of a grate (Figure 1).

Ginning occurs at the point $-A$, where the cotton particles impact to the surface of the grate. Here, an impact pair is a combination of two parts of the system: "cotton particle + stationary grate of the working chamber". The interaction of system links may be accompanied not by central but by oblique impacts 


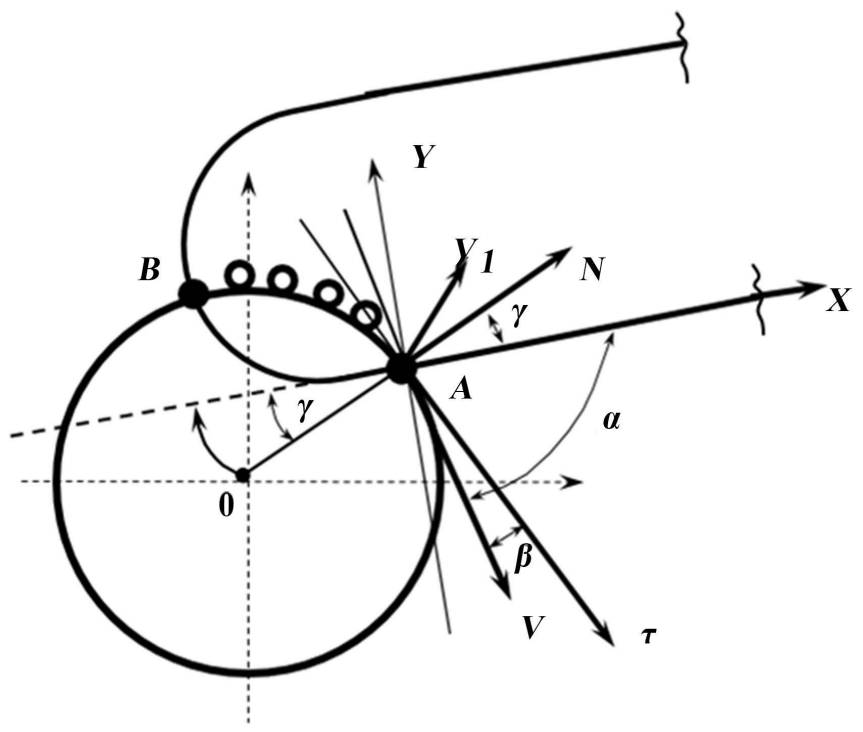

Figure 1. Scheme of determining cotton fiber from seeds at the ginning point.

[5] [6]. The consideration of such regimes, in addition to using the coefficient $\mathrm{K}$-restoration of the impact velocity normal to the surface of the grate, requires additional assumptions, that is, to evaluate the change in the tangential component of this velocity. When calculating such a system taking into account oblique impacts, two hypotheses are used, conventionally called: viscous and dry friction during oblique impact.

Let us consider the cotton particle as a material point of mass $m$, feeding at an angle $\alpha$ to the non-moving plane of the grate. Velocity of the cotton particle before and after impact: respectively $V$ and $V_{1}$. Then the velocity components $V$, $V_{1}$ along the axes $A X$ and $A C$ will be:

$$
\left\{\begin{array}{l}
V_{x}=V_{\sin \alpha} \\
V_{y}=V_{\cos \alpha}
\end{array},\left\{\begin{array}{l}
V_{1 x}=V_{1 \sin \beta} \\
V_{1 y}=V_{1 \cos \beta}
\end{array}\right.\right.
$$

According to both hypotheses, the normal components $-V_{y}, V_{1 y}$ speeds before and after the impact are related by the expression.

$$
V_{1 y}=K V_{y}
$$

The tangential components $V_{l x}, V_{x}$-according to the hypothesis of viscous friction are related.

$$
V_{1 x}=(1-\lambda) V_{x}
$$

where $\lambda$ is the coefficient of instantaneous friction upon impact.

Equations (2) and (3) - establish a linear relationship between before and after impact velocity components.

According to (3), the tangential component of the impact pulse, $m \cdot \lambda \cdot V_{x}$, does not depend on the magnitude of its normal component and is determined by the coefficient $\lambda$, which depends on the properties of the surface of the grate and cotton particle, as well as the relative sliding speed, just as the resistance 
force under viscous friction is proportional to the cotton particle motion speed.

According to the hypothesis of "conditional viscous friction", the angle of reflection of the cotton particle from the grate $-\beta$ is determined by the following dependence:

$$
\operatorname{tg} \beta=\frac{1-\lambda}{K} \operatorname{tg} \alpha
$$

According to the hypothesis of "conditional dry friction", the tangential component of the impact pulse is proportional to its normal component. In this case, the coefficient of proportionality is equal to the coefficient $-f$ dry friction:

$$
V_{1 x}-V_{x}= \pm f\left(V_{1 y}-V_{y}\right)
$$

The sign "+" or "-" is chosen opposite, to the sign $V_{x}$, taking into account the fact that impact interactions always lead to a decrease in the relative velocity of the cotton particle along the plane of the grate.

In this case, the angle of reflection $-\beta$ (cotton particle) is determined by the following dependence:

$$
\operatorname{tg} \beta=\frac{1}{K} \operatorname{tg} \alpha-f\left(1+\frac{1}{K}\right)
$$

If the condition:

$$
\operatorname{tg} \alpha<f(1+K)
$$

is fulfilled, then the cotton particle does not slip along the plane, but a rebound occurs. In this case, gin performance will decrease. Provided:

$$
\operatorname{tg} \alpha \geq f(1+K)
$$

productivity increases due to the strong adhesion of the cotton particle along the teeth of the saw when impacting along the grate.

According to the first hypothesis, the tangential and normal components of the pulse force are determined by the following dependence:

$$
\begin{aligned}
& S_{\tau}=m(2-\lambda) V \sin \alpha \\
& S_{N}=-m(1+K) V \cos \alpha
\end{aligned}
$$

And according to the second hypothesis:

$$
\begin{aligned}
& S_{\tau}=m \cdot V \sin \alpha \cdot f\left(K \frac{\cos \beta}{\cos \alpha}-1\right) ; \\
& S_{N}=-m \cdot V \cos \alpha\left(K \frac{\cos \beta}{\cos \alpha}-1\right) ;
\end{aligned}
$$

Figure 2 shows the changes in the angle of reflection $-\beta$ as a function of $-\alpha$ under the "conditionally viscous" hypothesis.

\section{The Study of the Regularity of Movement of a Cotton Piece on a Grid Surface}

The principles of movements of the cotton particle in the horizontal $(A B)$ part of 


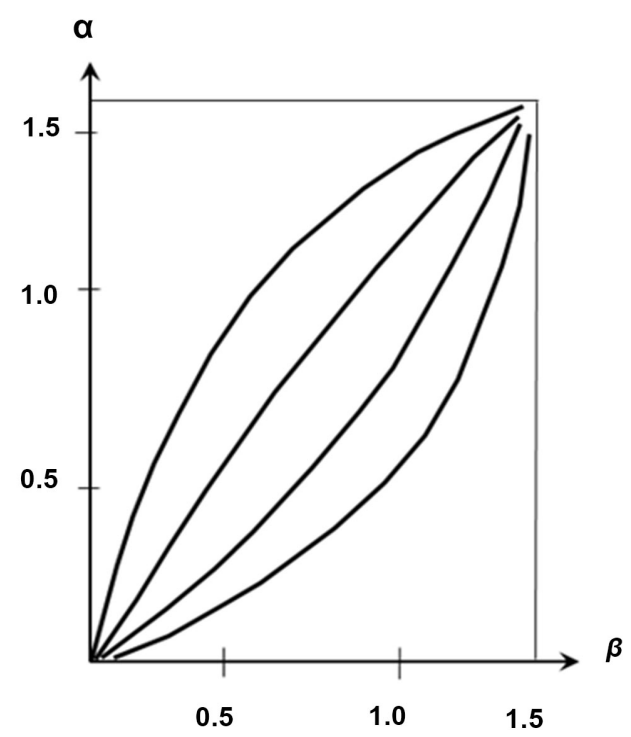

Figure 2. Changes in the angle of reflection $-\beta$ as a function of $-\alpha$ under the "conditionally viscous" hypothesis.

the newly constructed ginning camera, (1) which is created by the authors (Figure 3).

In the process of ginning, cotton particles that are separated from cotton seeds will be sent afresh to the ginning camera. In this process cotton seed parts that have fibers move through the $A B$ part of the construction (1). During the movement of cotton particles get affected by pressing to various surface (3) and forceful turbulent and horizontal movements of the surface. Some foreign mixtures and additional unnecessary objects get separated by the turbulence of the various surfaces and movement of the paneled (4) stripes. Ginning efficiency and the quality of the cotton will be improved as the defects of the cotton particles are removed. Figure 4 shows the mathematical model of the studying problem.

"Cotton particle + net surface" movement principle of the Cartesian coordinate system, we shall examine based on the raws of Cartesian coordinate system [7] [8] [9]. Forces that influence to his system:

$F_{0}(t)=A_{0} \sin \omega_{0} t$-harmonic outsider force that affect the various surface;

$F_{1}=$ const - paneled planks force that affects to the cotton seeds with fiber;

$F_{2}=F_{x}=f_{1} P=f_{1} m g-A X$ inertial power by the panel;

$F_{3}=f_{2} * F_{1}$-inertial force around the plank;

$P=m g-$ force of the weight of the cotton particle;

$f_{1}, f_{2}$-inertial coefficients.

Principles of the examined movements

$$
\left\{\begin{array}{l}
\frac{\mathrm{d} x}{\mathrm{~d} t}+K_{1} X=F_{1} \cos \alpha-F_{x}+A_{0} \sin \omega_{0} t \\
\frac{\mathrm{d} y}{\mathrm{~d} t}+K_{2} Y=F_{1} \sin \alpha+N-P+F_{3} \cos \alpha
\end{array}\right.
$$




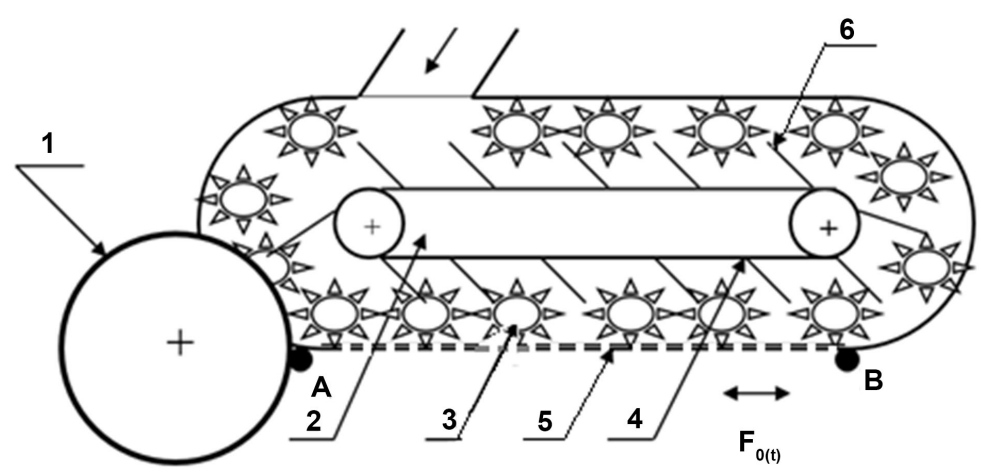

Figure 3. Scheme of movement of a piece of cotton on a grid surface. 1-Saw sylinder, 2-belt conveyor, 3-cotton particle, 4-belt, 5-grate, 6-belt strip.

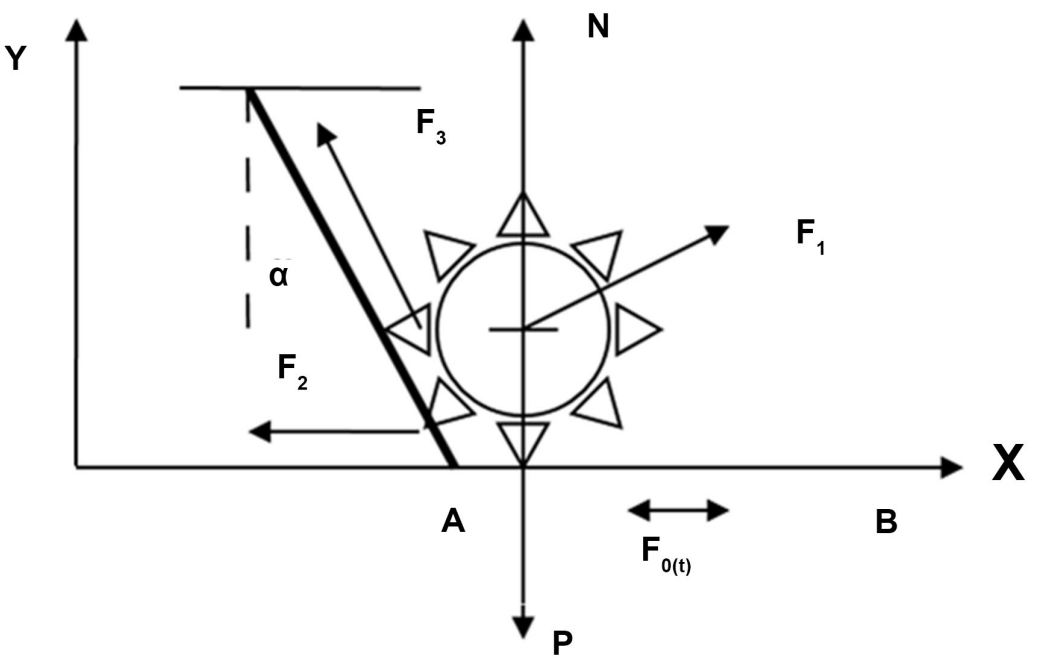

Figure 4. Scheme for determining the effect of force for moving a single piece of cotton on a grid surface.

Here: $K_{1}, K_{2}$-elastic coefficients of cotton particle by horizontal and vertical movements;

$N$-pressing force of the cotton seeds with power to the various surface;

$a$-the angle that is created by the paneled plank with vertical, grade.

By dividing all sides of the system (1) by $m$ weight, we shall have the following second ordered multiple gender differential formula:

$$
\left\{\begin{array}{l}
\ddot{X}+\omega_{1}^{2} X=\frac{F_{1}}{m} \cos \alpha-\frac{F_{x}}{m}+\frac{A_{0}}{m} \sin \omega_{0} t \\
\ddot{Y}+\omega_{2}^{2} Y=\frac{F_{2}}{m} \sin \alpha+\frac{N-P}{m}+\frac{F_{y}}{m} \cos \alpha
\end{array}\right.
$$

If we consider that movements of the particles of the cotton particle are done on various surfaces, we can find the normal pressing power from the second (14) formula.

$$
\begin{gathered}
Y=0 ; \\
N=P-F_{1} \sin \alpha-F_{3} \cos \alpha ;
\end{gathered}
$$


In this case, consider the following equation:

$$
X+\omega_{1}^{2} \ddot{X}=\frac{F_{1}}{m} \cos \alpha-\frac{F_{X}}{m}+\frac{A_{0}}{m} \sin \omega_{0} t ;
$$

The general solution of the homogeneous differential equation is found in:

$$
X=e^{k t} \text {; }
$$

$(17) \rightarrow(16):$

$$
\begin{gathered}
\ddot{X} e \omega_{1}^{2} \frac{X}{1}=0 \rightarrow k^{2}+\omega_{1}^{2}=0 ; \\
K_{1}=-\sqrt{\omega_{1} \cdot i} ; \\
K_{2}=+\sqrt{\omega_{1} \cdot i} ;
\end{gathered}
$$

General solution:

$$
X(t)=C_{1} \cos \omega_{1} t+C_{2} \sin \omega_{1} t
$$

Basic conditions:

$$
X(t)=0 \rightarrow\left\{\begin{array}{l}
X(0)=0 \\
\dot{X}(0)=V_{0}
\end{array}\right.
$$

$(22) \rightarrow(21)$

$$
\begin{gathered}
C_{1}=0 \\
C_{2} \omega_{1}=V_{0} ; \quad C_{2}=\frac{V}{\sqrt{\omega}} ;
\end{gathered}
$$

Means: regularity of private fluctuation:

$$
X_{1}(t)=\frac{V_{0}}{\omega_{1}} \sin \omega_{1} t ;
$$

Consider a non-homogeneous differential equation of the second degree:

$$
\ddot{X}+\omega_{1}^{2} X=\frac{F_{1}}{m} \cos \alpha-\frac{F_{X}}{m} ;
$$

If we denote,

$$
\frac{F_{1}}{m} \cos \alpha-\frac{F_{x}}{m}=A
$$

That:

$$
\ddot{X}+\omega_{1}^{2} X=A ;
$$

( $A=$ const. $)$

We find solution (28) at $X=B$.

Then:

$$
\omega_{1}^{2} \cdot B=A \rightarrow B=\frac{A}{\omega_{1}^{2}}=\frac{1}{\omega_{1}^{2} m}\left(F_{1} \cos \alpha-F_{x}\right) ;
$$

So: 


$$
X_{2}(t)=B
$$

Consider a non-homogeneous differential equation of the second degree:

$$
\ddot{X}+\omega_{1}^{2} X=\frac{A_{0}}{m} \sin \omega_{0} t
$$

Solution:

$$
X(t)=C \cdot \sin \omega_{0} t
$$

$(32) \rightarrow(31) \rightarrow$

$$
\begin{aligned}
& \omega_{0}^{2} C+\omega_{1}^{2} C=\frac{A_{0}}{m} ; \\
& C=\frac{A_{0}}{m\left(\omega_{1}^{2}-\omega_{0}^{2}\right)} ;
\end{aligned}
$$

Means: we found the pattern of movement of forced oscillations, that is:

$$
X_{3}(t)=\frac{A}{m\left(\omega_{1}^{2}-\omega_{0}^{2}\right)} \cdot \sin \omega_{0} t ;
$$

We have the principle of the general movement of the "cotton particle + net surface" or

$$
\begin{gathered}
X(t)=X_{1}(t)+X_{2}(t)+X_{3}(t) \\
X(t)=\frac{V_{0}}{\omega_{1}} \sin \omega_{1} t=\frac{A_{0}}{m\left(\omega_{1}^{2}-\omega_{0}^{2}\right)} \sin \omega_{1} t+F_{1} \cos \alpha-\frac{F_{x}}{m \omega_{1}^{2}}
\end{gathered}
$$

It can be seen from solution (37) that the system has a pattern of forced translational harmonious movement.

The results of these studies can be used in the design of fiber separation machines of various designs [9] [10] [11].

\section{Conclusions}

Selecting the value of the frequency of the particular vibration- $\omega_{1}$ and the vibration frequency of the fiber- $\omega_{0}$, it is possible to ensure the movement of cotton particles on the net surface in a loosened form and it is possible to achieve the removal of various foreign matters.

It is also possible to ensure the probability of falling bare cotton seeds from the net surface.

As a result of the study, the following results were obtained:

a) The law of the movement of cotton particles in the ginning zone was studied, on the basis of which mathematical equations were obtained;

b) The normal and tangential components of the force of impulse impact of the cotton particle from the grate are determined;

c) The law of the movement of the cotton particles on the mesh surface of the genie is studied;

d) The movement of cotton particle under the action of the plank of the belt conveyor was studied; 
e) Studies have been conducted to solve the mathematical equations obtained as a result of the study.

\section{Confirmation}

In this manuscript, the law of motion of a single cotton particle in the ginning zone and the law of motion of incompletely separated cotton particles from point $\mathrm{A}$ to point $\mathrm{B}$ on the mesh surface of the new working camera of the saw gin were studied that offered and patented by the authors. The equations of motion are obtained. This process occurs until the fiber is completely separated from the seeds, and the peeled seeds from the fiber leave through the mesh surface for the next technological process. Then the fiber is pressed after cleaning.

\section{Conflicts of Interest}

The authors declare no conflicts of interest regarding the publication of this paper.

\section{References}

[1] Studensev, A.I. (1929) Raw Material Roller of Saw Gin. Abstract Collection Cotton Business, 10, 10-15. (In Russian)

[2] Kotov, D.A. and Boldinsky, G.I. (1964) About the Allocation of Seeds from the Seed Roller of the Saw Gin. Collection of Scientific Papers (Tashkent Textile Institute), 18, 67-76. (In Russian)

[3] Tillyaev, M.T. (1974) Investigation of the Influence of the Seed Roller Rotation Accelerator on the Main Indicators of the Ginning Process. Candidate of Technical Science Dissertation, Tashkent, 48-64. (In Russian)

[4] Tyutin, P.N. (1967) Some Issues Regarding the Quality of Sharpening of the Gin Saws. Candidate of Technical Science Dissertation, Tashkent, 56-59. (In Russian)

[5] Lensky, A.N. and Loboda, V.M. (1972) Modeling Contact Interactions of Bodies in Impact Systems. Mechanics of Machines. Science, monography, 33-34. (In Russian)

[6] Nagoev, R.F. (1967) Correct Impulse Motion in a One-Dimensional System. Applied Mechanics of Machine, tutorial, 31-32. (In Russian)

[7] Piskunov, N.S. (1985) Differential and Integral Calculation, Science, Tutorial, 251254. (In Uzbek)

[8] Burnashev, R.Z., Sarimsakov, O.S. and Muradov, R. (2001) The Dynamic Problem of the Interaction of Raw Cotton with the Working Bodies of Processing Machines. The Journal Problem of Mechanics, 3/4, 55-58.

[9] Sarimsakov, O.S., Ergashev, J., Ergashev, S. and Kayumov, A. (1991) Working Chamber of the Saw Fiber Separator. Copyright Certificate (Patent) of the SU. No1680811. Bulletin of Inventions, Moscow, No. 36.

[10] Ergashev, S.T., Sarimsakov, O.S., Kurbanov, R.N. and Burnashev, R.Z. (1991) The Working Chamber of the Saw Fiber Separator. Copyright Certificate (Patent) of the SU. No. 1693140. Bulletin of Inventions, Moscow, No. 43.

[11] Akhmedhodjaev, X., Muradov, R., Sarimsakov, O.S. and Kayumov, A. (1991) Fiber Separator. Copyright Certificate (Patent) of the SU. No. 1622432. Bulletin of Inventions, Moscow, No. 3. 